\title{
COMMENTARY
}

\section{Induced pluripotent stem cell-derived neural stem cells: new hope for stroke?}

Jia Liu

See related research by Yuan et al., http://stemcellres.com/content/4/3/73

\begin{abstract}
Human induced pluripotent stem cells (iPSCs) have attracted increasing interest in the field of ischemic stroke therapy, due to the lack of ethical concerns and reduced risk of immune rejection. However, the safety and efficiency of the donor iPSC derivates in the ischemic brain challenged the therapeutic efficacy of iPSC transplantation. Studies have focused on improving the methods to induce neural derivates from iPSCs and on trying to determine how these cells and the host ischemic environment influence each other. A recent study by Yuan and colleagues reported that neural stem cells induced from human iPSCs using retinoic acid and serum-free medium showed stable neural phenotype. After acute transplantation into the ischemic stroke model, these cells survived, migrated into the ischemic penumbra, differentiated into mature neural cells and showed beneficial effects on functional recovery. Their findings take a clear step towards the clinical application of human iPSCs for ischemic stroke. A wide range of careful studies may be raised from their work, from basic research to preclinic, to develop the best therapy for ischemic stroke.
\end{abstract}

Stroke is the leading cause of death and disability among the adult population around the world. Ischemic stroke comprises the majority, accounting for $80 \%$ of the total strokes. Recent advances have improved stroke management, while current therapeutic strategies offer only modest effects. Alternatively, stem cell-based therapies using a variety of embryonic, fetal and adult stem cells have been investigated as potential regenerative and/or protective treatments for ischemic stroke over the last

Correspondence: jialiu2013@yeah.net

Department of Neurobiology, Care Sciences and Society, Karolinska Institutet, 5th floor, Novum, SE-141 86, Stockholm, Sweden two decades, with encouraging functional improvements in animal experiments. However, these types of stem or progenitor cells raise ethical issues and inevitably are genetically dissimilar to the recipient with potential risk of immune rejection. The creation of induced pluripotent stem cells (iPSCs) through reprogramming of somatic cells has revolutionized cell therapy by providing a source of autologous cells for transplantation. These cells could be obtained from the stroke patients, avoiding ethical concerns and reducing the need for immunosuppression. A recent article by Yuan and colleagues [1] investigated a modified protocol to induce neural stem cells (NSCs) from human iPSCs, and the survival, migration and differentiation of NSCs and functional recovery of animals were evaluated after acute transplantation [1].

In preclinical stroke studies, the issues recommended by the Stroke Therapy Academic Industry Roundtable (for example, sample size, blinded evaluation of outcome) should be taken into consideration when designing and conducting the experiments and assessments $[2,3]$. In the work by Yuan and coworkers, the sample size for each experimental group was small (five cases/ group) [1], which may weaken the statistical power of the results. The authors stated that the experimental groups were divided randomly but did not describe how the randomization was achieved. Furthermore, they failed to perform the neurobehavioral tests blindly, which is extremely important for the scientific society to evaluate whether the results raised from the current study are solid enough to believe or not. A sample size calculation, an appropriate method for randomization and a blind functional assessment as recommended by the Stroke Therapy Academic Industry Roundtable are therefore desired in future studies [2].

Safety is one major issue in the preclinical cell therapy for stroke. Despite the lack of an ethical problem, the high teratoma-forming property of iPSCs is a critical problem. iPSCs might have greater risk for 
tumorigenesis than embryonic stem cells (ESCs) due to genetic and epigenetic aberrations [4]. Therefore, an optimal and efficient method to generate iPSC-NSCs is extremely crucial. Yuan and colleagues induced human iPSCs into NSCs using the combination of retinoic acid and serum-free medium to increase the efficiency and to avoid contamination from serum and other cells [1]. The induced NSCs showed stable neural phenotype and capacity of proliferation and differentiation into neural lineages, and the authors reported that no tumor was found in two transplanted rats after 3 months. Others have reported similar findings by dual inhibition of the SMAD pathway to induce iPSC-NSCs $[5,6]$.

Different induction methods may lead to differences in characteristics of iPSC-NSCs. Thus, it is worth comparing whether iPSC-NSCs generated through different protocols could lead to the same results under equivalent experimental conditions, and which protocol might be better to generate iPSC-NSCs for use in ischemic stroke. Current neural induction methods for iPSCs mainly depend on previous findings for ESCs. Although similar, ESCs and iPSCs display significant differences [7]. Thus, it is crucial to conduct careful and detailed investigations on the neural induction method for iPSCs. Noteworthy, in the work by Yuan and colleagues the observation period for tumor formation in the majority cases was short at only 2 weeks [1]. In one study with spinal cord injury, even the functional outcome was improved at an early stage within 6 weeks after human ESC-derived NSCs transplantation, but the tumor formation was found 12 weeks post-implantation [8], suggesting that the short duration may be not enough for the grafted cells to achieve overproliferation and therefore form a tumor. Yuan and colleagues further made an effort to conduct long-term observation on tumorigenesis of the grafted iPSC-NSCs, but it is hard to draw solid conclusions with the really small sample size of only two cases. Long-term studies with larger sample size on the tumorigenesis of iPSC-NSCs are thus needed . Furthermore, although Yuan and colleagues and others all demonstrated that no tumors were found in the ischemic brain $[1,5,6]$, the potential for tumor formation in other organs must be considered, particularly when a systemic delivery route of cells is chosen.

Before addressing the effects of iPSC-NSCs on the host, it is necessary to determine how the host environment affects the grafted cells. Yuan and colleagues reported that the transplanted iPSC-NSCs survived and differentiated into mature neurons and astrocytes in the ischemic brain [1]. This observation demonstrated that the host ischemic environment at least was not completely hostile to iPSC-NSCs. The authors further showed that grafted iPSC-NSCs migrated from the striatum toward the ischemic boundary, indicating it is possible to design less invasive ways to deliver cells instead of injecting cells directly into the injury site due to their migration property. One should note that current iPSC-NSC transplantation occurred during the acute stage. However, the derivation of human iPSCs and their derivates is still a month-long process that hardly fits with the stroke adage that 'time-is-brain'. Thus, to achieve autologous iPSC-NSC transplantation for stroke patients, preclinical studies of subacute and chronic transplantation are needed.

The central issue of cell therapy is whether the grafted cells could exert beneficial effects to the host. Yuan and colleagues observed significantly improved functions in grafted rats 3 weeks after transplantation [1], which provided valuable evidence for the clinical application of human iPSC-NSCs in ischemic stroke. However, the authors did not determine the potential mechanism leading to the functional recovery. One major feature of ischemic stroke caused by large vascular occlusion is the significant reduction of cerebral blood flow in the infarct core and surrounding area. The ischemic penumbra has been defined as a hypoperfused area around the ischemic core that is potentially reversible with a timely intervention. Murine NSCs derived from fetal central nervous tissues were reported to release elevated levels of proangiogenic factors such as vascular endothelial growth factor $[9,10]$ and nitric oxide [11], and to promote angiogenesis with increased cerebral blood flow in the ischemic boundary region [12]. Therefore, one reasonable hypothesis is that human iPSC-NSCs might be able to restore cerebral blood flow in the ischemic penumbra through angiogenesis. In addition, human iPSCNSCs might also exert other effects on the penumbra tissues, such as rescue of apoptotic cells, modulation of stroke-induced inflammation and enhancement of intrinsic repair mechanism. Further studies are urgently needed to understand the possible mechanisms behind the functional recovery and to provide reliable and strong evidence for translating iPSC-based cell therapy into the clinical setting.

The article by Yuan and colleagues has provided the scientific community with a lot to think about, and, more importantly, a number of doable studies may result from their work. The final goal of cell therapy research is to provide the maximum therapeutic effects to patients with the least amount of suffering and risk. Therefore, the development of autologous iPSC-NSC treatment requires huge efforts, continual accumulations of major findings and careful studies on multiple aspects, which will contribute to achieving the best therapy.

\section{Abbreviations}

ESC: embryonic stem cell; iPSC: induced pluripotent stem cell; NSC: neural stem cell. 


\section{Competing interests}

The author declares that she has no competing interests.

Published: 25 September 2013

\section{References}

1. Yuan T, Liao W, Feng NH, Lou YL, Niu X, Zhang AJ, Wang Y, Deng ZF: Human induced pluripotent stem cell-derived neural stem cells survive, migrate, differentiate, and improve neurological function in a rat model of middle cerebral artery occlusion. Stem Cell Res Ther 2013, 4:73.

2. Fisher M, Feuerstein G, Howells DW, Hurn PD, Kent TA, Savitz SI, Lo EH: Update of the stroke therapy academic industry roundtable preclinical recommendations. Stroke 2009, 40:2244-2250.

3. Stroke Therapy Academic Industry Roundtable (STAIR): Recommendations for standards regarding preclinical neuroprotective and restorative drug development. Stroke 1999, 30:2752-2758.

4. Tsuji O, Miura K, Okada Y, Fujiyoshi K, Mukaino M, Nagoshi N, Kitamura K, Kumagai G, Nishino M, Tomisato S, Higashi H, Nagai T, Katoh H, Kohda K, Matsuzaki Y, Yuzaki M, lkeda E, Toyama Y, Nakamura M, Yamanaka S, Okano $\mathrm{H}$ : Therapeutic potential of appropriately evaluated safe-induced pluripotent stem cells for spinal cord injury. Proc Natl Acad Sci U S A 2010, 107:12704-12709.

5. Mohamad O, Drury-Stewart D, Song M, Faulkner B, Chen D, Yu SP, Wei L: Vector-free and transgene-free human iPS cells differentiate into functional neurons and enhance functional recovery after ischemic stroke in mice. PLoS One 2013, 8:e64160.

6. Polentes J, Jendelova P, Cailleret M, Braun H, Romanyuk N, Tropel P, Brenot M, Itier V, Seminatore C, Baldauf K, Turnovcova K, Jirak D, Teletin M, Come J, Tournois J, Reymann K, Sykova E, Viville S, Onteniente B: Human induced pluripotent stem cells improve stroke outcome and reduce secondary degeneration in the recipient brain. Cell Transplant 2012, 21:2587-2602.

7. Puri MC, Nagy A: Concise review: Embryonic stem cells versus induced pluripotent stem cells: the game is on. Stem Cells 2012, 30:10-14.

8. Sundberg M, Andersson PH, Akesson E, Odeberg J, Holmberg L, Inzunza J, Falci S, Ohman J, Suuronen R, Skottman H, Lehtimaki K, Hovatta O, Narkilahti S, Sundstrom E: Markers of pluripotency and differentiation in human neural precursor cells derived from embryonic stem cells and CNS tissue. Cell Transplant 2011, 20:177-191.

9. Harms KM, Li L, Cunningham LA: Murine neural stem/progenitor cells protect neurons against ischemia by HIF-1alpha-regulated VEGF signaling. PLoS One 2010, 5:e9767.

10. Madhavan L, Ourednik V, Ourednik J: Neural stem/progenitor cells initiate the formation of cellular networks that provide neuroprotection by growth factor-modulated antioxidant expression. Stem Cells 2008, 26:254265.

11. Wang L, Shi J, van Ginkel FW, Lan L, Niemeyer G, Martin DR, Snyder EY, Cox NR: Neural stem/progenitor cells modulate immune responses by suppressing T lymphocytes with nitric oxide and prostaglandin E2. Exp Neurol 2009, 216:177-183.

12. Jiang $Q$, Zhang $Z G$, Ding GL, Zhang $L$, Ewing JR, Wang L, Zhang R, Li L, Lu M, Meng H, Arbab AS, Hu J, Li QJ, LiPourabdollah Nejad DS, Athiraman H, Chopp M: Investigation of neural progenitor cell induced angiogenesis after embolic stroke in rat using MRI. Neuroimage 2005, 28:698-707.

doi:10.1186/scrt326

Cite this article as: Liu: Induced pluripotent stem cell-derived neural stem cells: new hope for stroke?. Stem Cell Research \& Therapy 2013 4:115. 